\title{
Thread Risk Modelling
}

\author{
Martin Knobloch \\ OWASP Netherlands, Netherlands \\ martin. knobloch@owasp.org
}

How secure must an application be? To take the appropriate measures we have to identify the risks first and think about the measures later. Threat risk modelling is an essential process for secure web application development. It allows organizations to determine the correct controls and to produce effective countermeasures within budget. This presentation is about how to do a Tread Risk Modelling. What is needed to start and where to go from there! 